\title{
The Design of Wireless Communication System Based on STC12+CC1101
}

\author{
Zhijian Yin ${ }^{1, ~ a ~}$, Kun Xiang ${ }^{2, b}$,Wang Fan ${ }^{3, c}$ \\ Jiangxi Science \& Technology Normal University, Nanchang, China \\ a86412656@qq.com, b1244689313@qq.com, '1245905084@qq.com
}

Keywords: Wireless communication; Automatic identification; CC1101

\begin{abstract}
Wireless data transmission in short distance is a kind of technology instead of cable, which is widely used in many current fields. And it solved the problem that it is difficult for cable wiring due to the limit of environment and condition. For the problem that the length of control command is different and the launch module cannot judge the emission data in the wireless communication of controlling the LED screen display, this paper designs a wireless communication system which can automatically identify the command length and send it to the receiving equipment by wireless communication. After consulting documents and summarizing predecessors' experience, this paper provides the overall design scheme of wireless communication system which can automatically identify the command length. And the wireless data transceiver module consists of CC1101 wireless RF chip produced by TI enterprise and STC12LE2052AD produced by STC enterprise. Through testing and performance debugging, the system can send the command whose length is arbitrary to the receiver by wireless communication.
\end{abstract}

\section{Introduction}

Wireless communication technology is one of the most popular research directions in the current field of information technology, and sensor technology, embedded technology, network technology and it consist of the important part of information IoT(Internet of Things) construction today. The most common wireless information interaction system includes the two parts, transmitting end and receiving end. This design uses CC1101 as the wireless communication module and the data are processed by STC12LE2052AD. Through testing and performance debugging, the system can send the command whose length is arbitrary to the receiver by wireless communication.

\section{The Design of System Hardware}

According to the requirements of system design, the design block diagram of system hardware is as follows in Fig. 1.

Working principle of the system: this system is a unit module circuit, which can not only be used as signal emission part, but also be signal receiver. The launch principle is that firstly single chip microcomputer(SCM) receives the host data by the serial port and then sends it to launch chip, and lastly the launch chip sends it out. The receiving principle is that wireless chip sends the received data to SCM, and SCM sends it to the host by the serial port. The working principles of all hardware circuits in this system are as follows. 


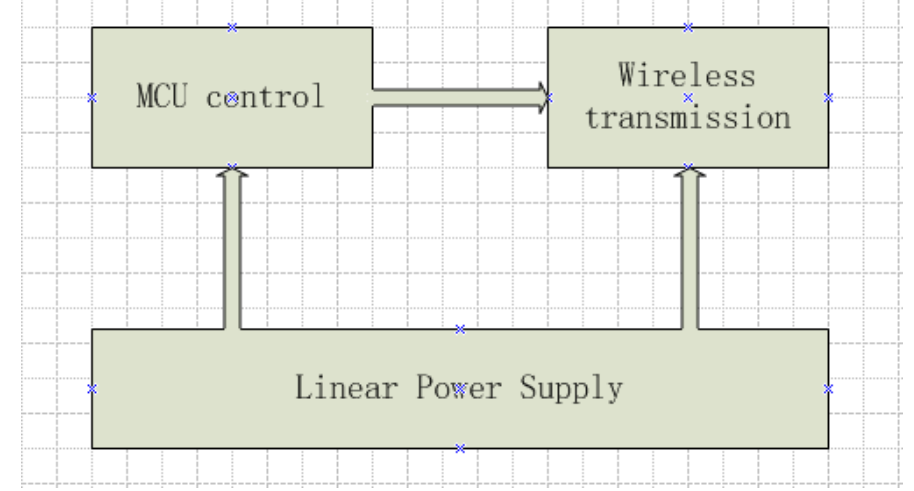

Fig. 1 The design block diagram of system hardware

Power Part. The regulated part of power supply adopts LM1117-3.3, and this is a voltage regulator series

with low dropout voltage and its stable output voltage is $3.3 \mathrm{~V}$. When the load current is $1 \mathrm{~A}$, the voltage drop is $1.2 \mathrm{~V}$. And the chip provides current limitation and thermal protection. The circuit contains a band gap reference voltage with Zener adjustment to ensure the accuracy of output voltage within $\pm 1 \%$.Its unit module circuit is shown in Fig. 2 below. C16, C17 and C18 are power filter capacitance, connected with the power supply of the chip, which are used to reduce the power supply ripple.
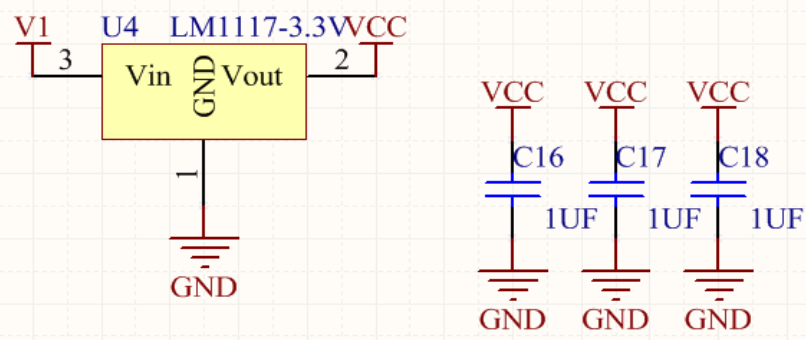

Fig. 2 Voltage regulator circuit

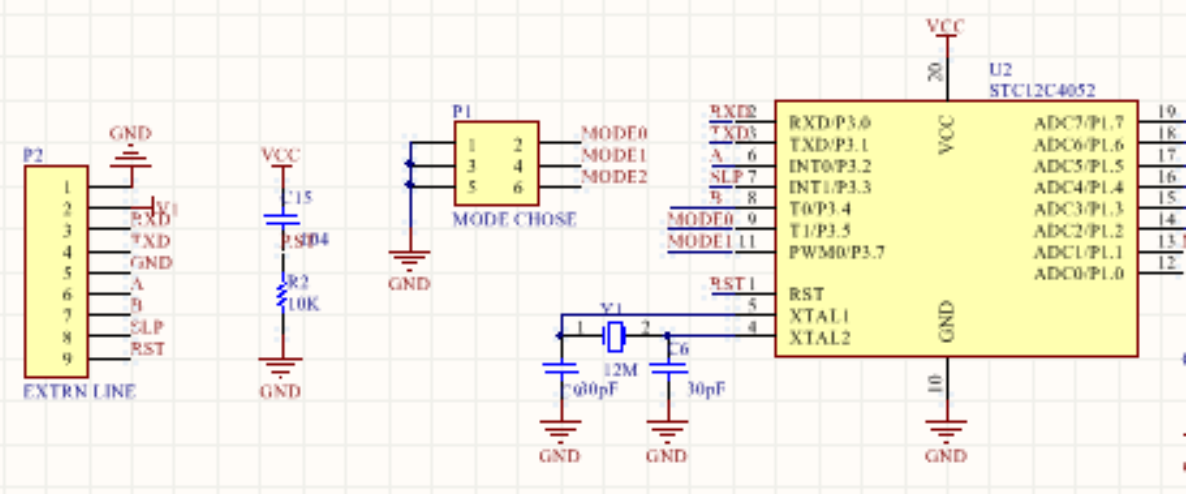

Fig. 3 The circuit of control part

Control Circuit Part. The control chip of the system is STC12LE4052 which is low-power SCM and is produced by Acer. The circuit of control part is shown in Fig. 3. P2 is used as interface circuit and the whole circuit module can be connected with other circuits for communication work. The reset circuit consists of C15 and R2, which makes the control chip reset when power every time. P1 is used as module selection circuit, which can control the communication channel of the system and make the multiplex circuits not interfere with each other when all they are working. The crystal oscillator unit circuit consists of Y1, C9 and C6, which provides oscillation signal for SCM.

Signal Receiving and Transmitting Circuit. The system chooses the CC1101 chip produced by TI, which is a high-performance RF transceiver with less than $1 \mathrm{GHz}$ frequency. And the design is intended for the application of extremely low-power RF, which is mainly used for industry, scientific research and medical treatment(ISM) and short range devices(SRD). CC1101 can provide wide hardware support of packet processing, data buffering, burst transmission, received signal 
strength indicator(RSSI), clear channel assessment(CCA), link quality indicator(LQI) and wake on radio(WOR). The system chooses $433 \mathrm{MHz}$ communication frequency band. The hardware principle diagram is shown in Fig. 4. The design of hardware circuit refers to the classic design scheme of TI, in which P3 is 50R matching antenna.

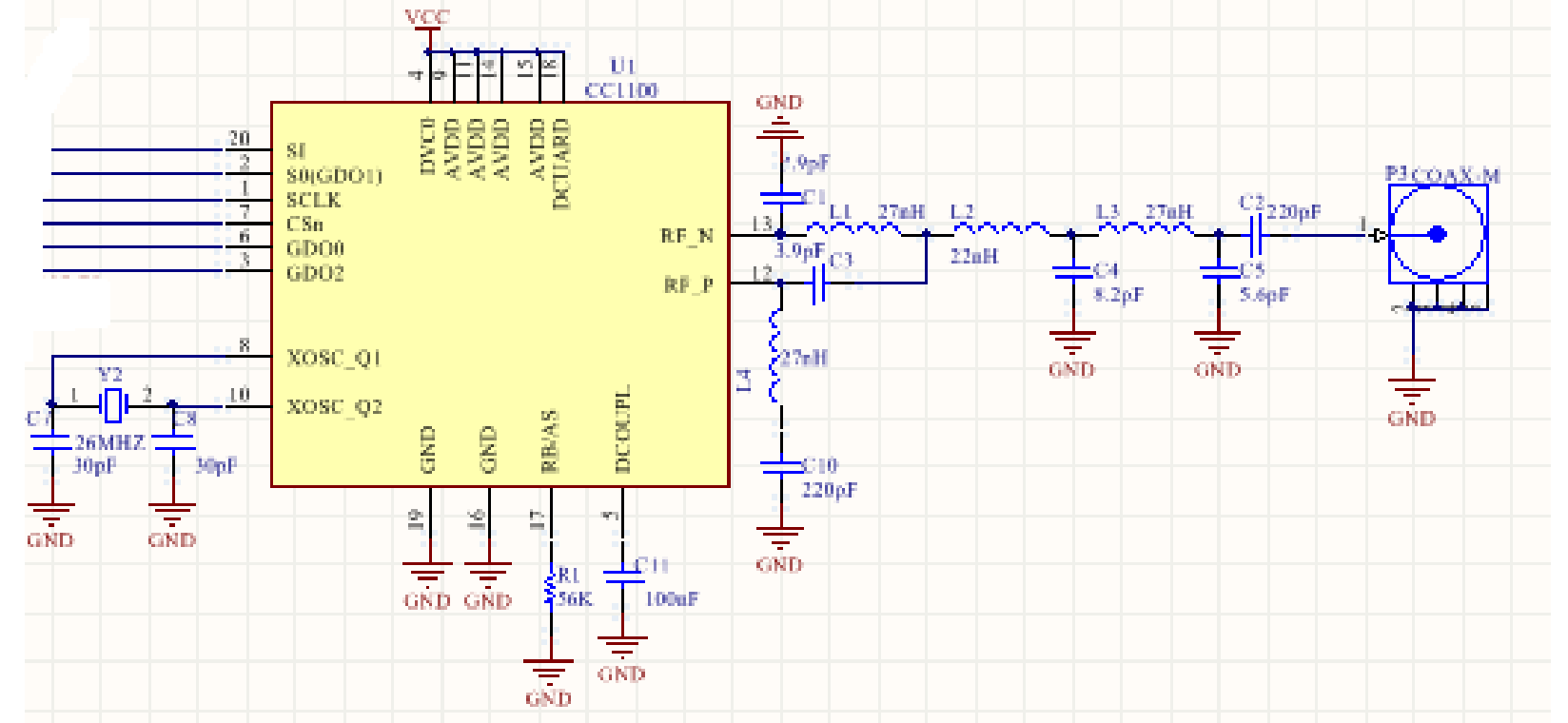

Fig. 4 Launch module circuit

\section{The Design of System Software}

The design of system software is completed in the software development platform of Keil uVision 4, and the program block diagram is shown in Fig. 5. The main function programs are as follows.

The Program of Channel Selection.

$$
\text { void channel_choose(void) }
$$

$\{$ XINDAO_CHANNEL=1;

$$
\begin{aligned}
& \text { if(XINDAO_CHANNEL) } \\
& \quad \text { halSpiWriteReg(CCxxx0_CHANNR, 0x00); }\}
\end{aligned}
$$$$
\text { if(!XINDAO_CHANNEL) }
$$

\}

$$
\{\text { halSpiWriteReg(CCxxx0_CHANNR, 0x01); }
$$

The communication channel selection is to control SCM to write a characteristic value to CCxxx0_CHANNR register of CC1101 to select the communication channel. The chip has 256 communication channels to be chosen and only two of them are chosen in this system design. 


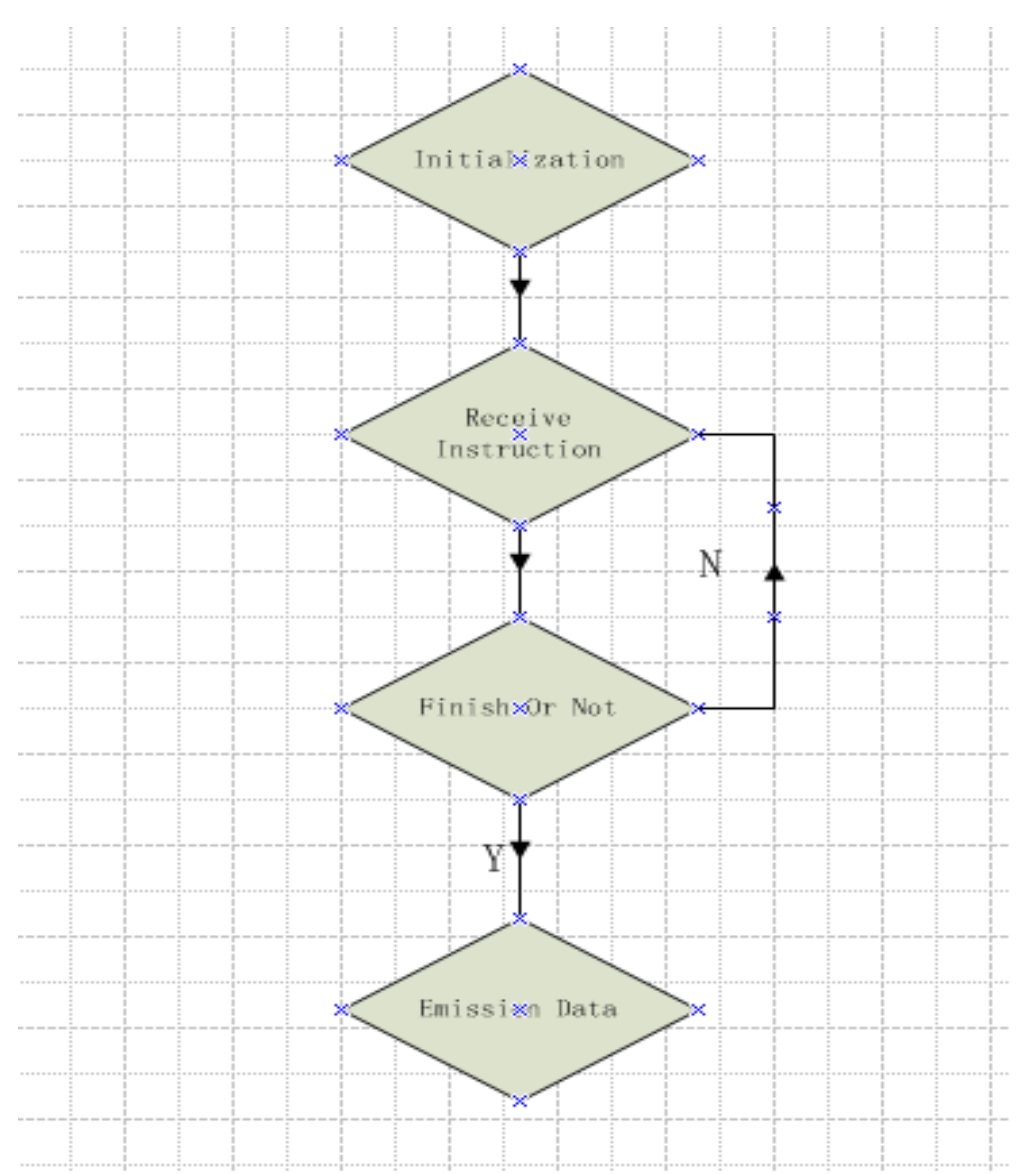

Fig. 5 The program block diagram

The Program of Character Length Recognition.

if(RI\&\&PC_rx)//Accepting 512 computer data

\{

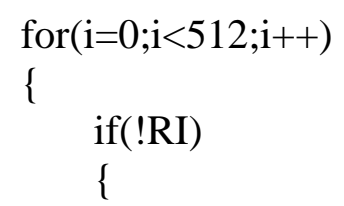


The basic principle of the program work is to determine the command length and whether the command is received completely by judging the length of time that RI is low level, and the system can automatically identify the data length(less than 512 bytes) from the serial port, and in this range, the system can send out these data by wireless communication regardless of how long the data sent by the host. It brings great convenience for users in circuit design, and it is free for the host to send data without considering the change of the data length and the host can send the commands according to the current actual need.

\section{The System Testing Result}

Test method: the system test chooses the software STC-ISP of Acer to have a passthrough communication test and the test block diagram is shown in Fig. 6. The communication data are sent via the serial port by the host 1 to the communication module 1 , and then the module 1 send it to the module 2, and lastly the module 2 sends the communication data to the host 2 by the serial port. In the testing results, it can been clearly seen that the received text data are in accord with emissive text data completely, which meets the initial requirements of the design.

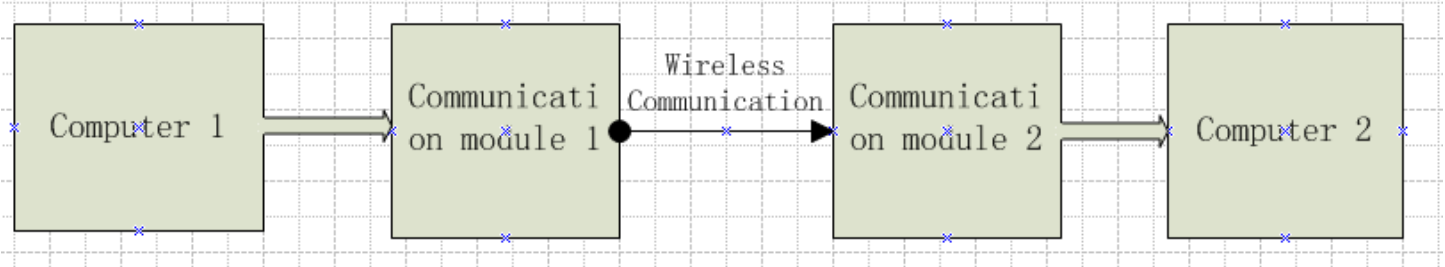

Fig. 6 The system testing block diagram

The physical diagram of the wireless communication module and the system testing results and are shown in Fig. 7, 8 respectively.

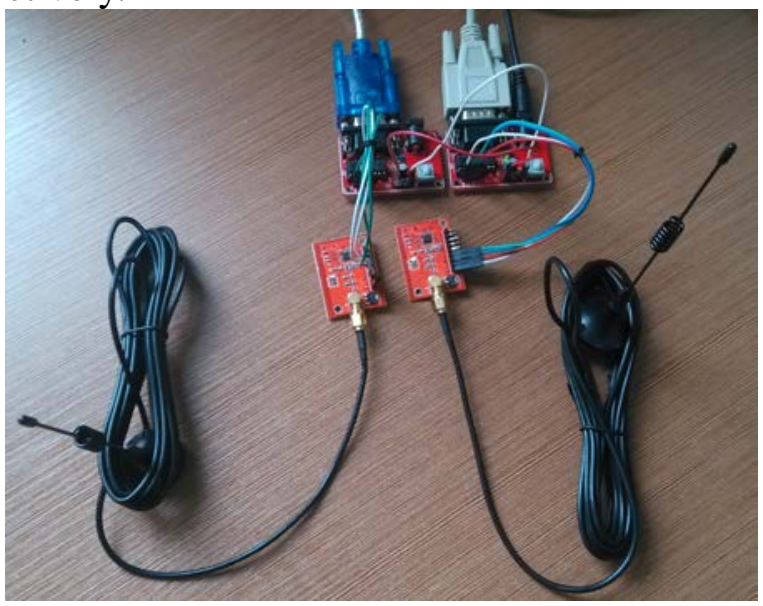

Fig. 7 The physical diagram of the wireless communication module 

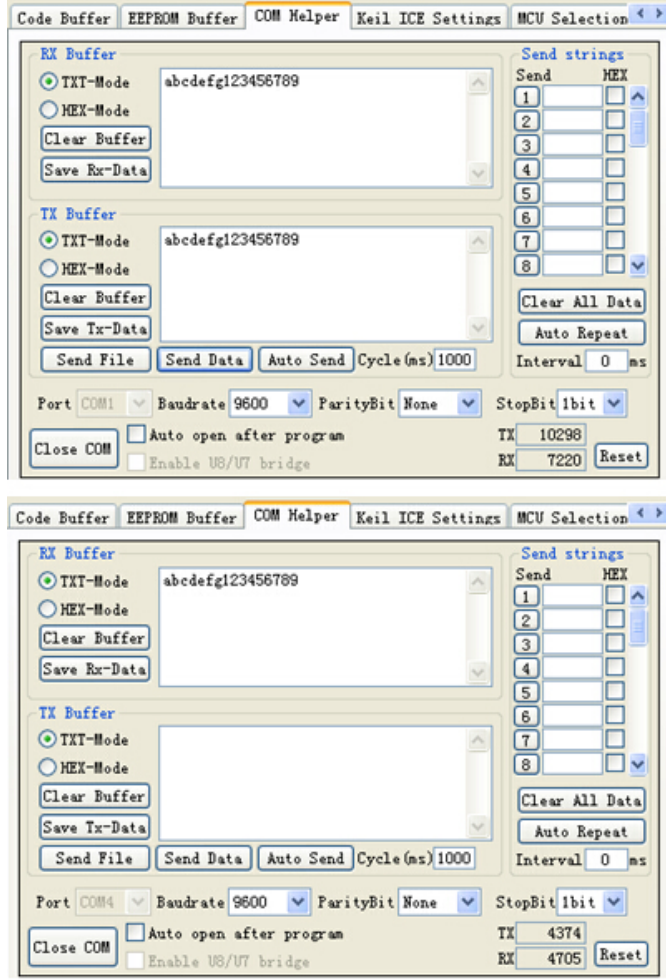
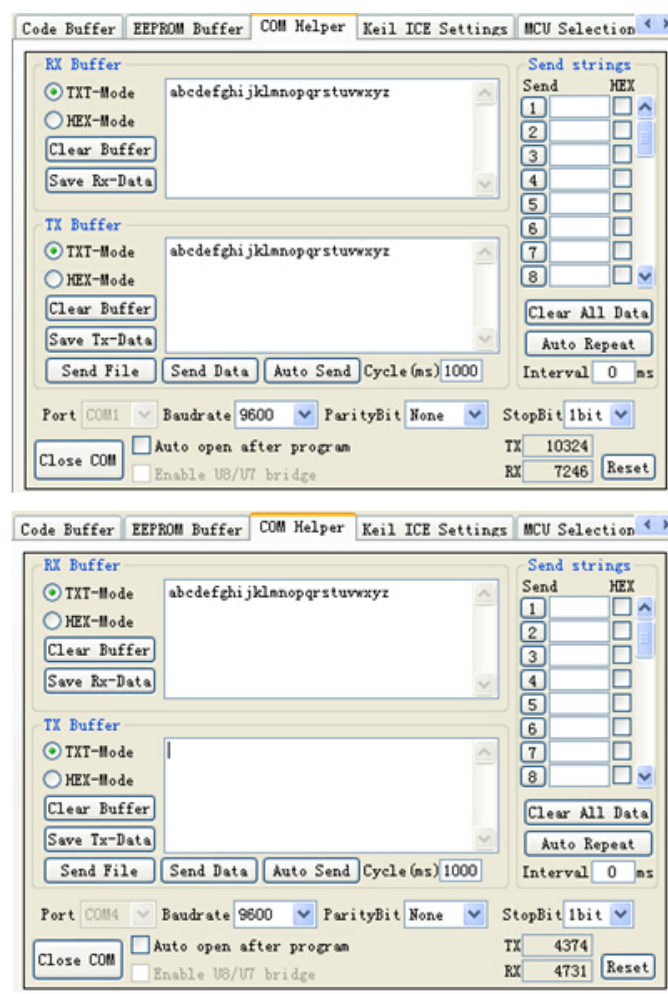

Fig. 8 The system testing results

\section{Summary}

This system can automatically identify the host command length and then send them to the receiver by the wireless communication after the actual test, which solved the problem that the command length is different in the LED synchronous display. And the host can send data command according to the current actual need. However, there is no doubt that there are some defeats to improve in this system, such as a certain code missing and the problem that it is difficult to receive and transmit large data. And with good components and software optimization, these problems will be further solved.

\section{Reference}

[1] Guiyou Cheng: Enhanced 8051 Microcontroller Practical Development Technology, Beijing, 2010.

[2] Lijun Li, Daihua Wang and Jing Zu: The Design of Wireless Data Transmission System Based on CC1100, Foreign Electronic Measurement Technology, 2007, 26(12): 42-45.

[3] Siliang Tan and Chaoqun Zou: Visual C++ Serial Communication Engineering Development Instance Navigation, Beijing, 2003.

[4] Limin Sun and Jianzhong Li: Wireless Sensor Network, Beijing, 2005.

[5] Wenzhong Li and Chaoyu Duan: ZigBee Wireless Network Technology Introduction and Actual Combat, Beijing, 2007. 